This is an Open Access article, distributed under the terms of the Creative Commons Attribution licence (http://creativecommons.org/licenses/by/4.0/), which permits unrestricted re-use, distribution, and reproduction in any medium, provided the original work is properly cited.

doi:10.1017/jfm.2017.274

\title{
Perturbation analysis of subphase gas and meniscus curvature effects for longitudinal flows over superhydrophobic surfaces
}

\author{
Darren G. Crowdy $\dagger$ \\ Department of Mathematics, Imperial College London, London SW7 2AZ, UK \\ (Received 30 October 2016; revised 27 March 2017; accepted 14 April 2017; \\ first published online 1 June 2017)
}

Integral expressions for the first-order correction to the effective slip length for longitudinal flows over a unidirectional superhydrophobic surface with rectangular grooves are determined under the assumptions that the meniscus curvature is small and the viscosity contrast between the groove-trapped subphase gas and the working fluid is significant. Both pressure-driven channel flows and semi-infinite shear flows are considered. Reciprocity ideas, based on use of Green's second identity, provide the integral expressions with integrands dependent on known flat-meniscus solutions found by Philip (Z. Angew. Math. Phys., vol. 23, 1972, pp. 353-372). The results extend earlier work by Sbragaglia \& Prosperetti (Phys. Fluids, vol. 19, 2007, 043603) on how weak meniscus curvature affects hydrodynamic slip. In particular, we derive a new integral expression for the first-order slip length correction due to weak meniscus curvature.

Key words: drag reduction, interfacial flows (free surface), microfluidics

\section{Introduction}

The study of superhydrophobic surfaces is an active area of research owing to its relevance in a rich variety of micro- and nano-fluidics applications. Such surfaces can dramatically reduce flow resistance in the manipulation of small volumes of fluid (Rothstein 2010). They typically have a surface microstructure that, owing to capillary effects, prevents fluid from fully penetrating interstitial regions between grooves, posts or protrusions leading to enhanced slip of the fluid over that surface. Consequently the usual assumption of a no-slip condition is no longer relevant (Neto et al. 2005; Lauga, Brenner \& Stone 2007) and must be replaced by some more accurate effective boundary condition allowing some degree of slip.

The focus of the present paper is on the case of surfaces made up of periodic arrays of unidirectional grooves where the flow along the surface is parallel to those structures (longitudinal flow). Ou, Perot \& Rothstein (2004) and Ou \& Rothstein (2005) have found in experiments for such longitudinal flows in microchannels that significant drag reduction can be achieved. Other experimental and numerical works,

$\dagger$ Email address for correspondence: d.crowdy@imperial.ac.uk 
of both longitudinal and transverse flow along grooved channels, have been carried out by Davies et al. (2006), Maynes, Webb \& Davies (2007b) and Woolford, Maynes $\&$ Webb (2009). Numerical investigations of drag reduction in this setting have been performed by Ng \& Wang (2009), Ng, Chu \& Wang (2010) and Teo \& Khoo (2010).

A common quantifier of the degree of slip is the so-called hydrodynamic slip length: for shear flows with shear rate $\dot{\gamma}$ over such a surface occupying the plane $y=0$ the velocity field $\boldsymbol{u}$ far from the plane of the surface takes the form

$$
\boldsymbol{u}=\dot{\gamma}(y+\lambda) \hat{\boldsymbol{x}}
$$

where $\hat{\boldsymbol{x}}$ is the flow direction. The constant $\lambda$ is the effective slip length and is a measure of the frictional properties of the surface: it is the fictional distance below the surface at which the shear flow would extrapolate to zero. Higher values of slip length mean greater slip at the surface. For pressure-driven channel flows the effective slip length $\lambda$ is defined by equating the mass flux through the channel to that which would ensue, for the same driving pressure gradient, if the superhydrophobic surface is replaced by a Navier-slip boundary with slip parameter $\lambda$.

Theoretical studies of superhydrophobic surfaces include that by Lauga \& Stone (2003) who harnessed analytical results due to Philip (1972) on solutions to a class of mixed boundary value problems for harmonic and biharmonic fields to infer their global slip properties. In their models the surfaces are made up of combinations of no-slip and no-shear surfaces so that the effect of a second viscous fluid enclosed in the grooves is ignored. Moreover Lauga \& Stone (2003) study arrangements in which all no-shear surfaces have the same curvature as the adjacent no-slip surfaces. Indeed the majority of existing theoretical and numerical studies adopt the same assumption that the meniscus does not protrude into or out of the fluid.

The purpose of the present paper is to offer theoretical insights on the situation in which both weak meniscus curvature and the effect of a viscous subphase fluid are taken into account. Concerning the effects on slip of a subphase gas, Davies et al. (2006), Maynes et al. (2007a) and Woolford et al. (2009) have carried out numerical simulations to study the effects of trapped pockets of air in closed-end grooves in unidirectional grooved surfaces and compared their results with experimental data. Ng \& Wang (2009) and Ng et al. (2010) devised numerical methods based on eigenfunction expansions to study the same problem, both in longitudinal and transverse flows, and including the incorporation of intrinsic slip on the solid-liquid surfaces. In an effort to model subphase gas effects theoretically Schönecker \& Hardt (2013) and Schönecker, Baier \& Hardt (2014) have proposed a semi-analytical method where the liquid-gas interface, taken to be flat, is assumed to be a constant non-zero shear-stress boundary which leads to a non-uniform local slip length distribution across the interface. Those authors produce approximate formulas for the effective slip length as a function of viscosity contrast and the flow geometry; the formulas are shown, by comparison with full numerical simulations, to give good approximations in many situations. Other models have been presented (Asmolov \& Vinogradova 2012; Nizkaya, Asmolov \& Vinogradova 2013, 2014) including a 'gas cushion model' which assumes that dissipation at the gas/liquid interface is dominated by the shearing of a continuous gas layer. The theoretical treatment relies on a so-called operator method (Nizkaya et al. 2014) which provides a mechanism which transplants the flow in the gas subphase to a local slip boundary condition at the liquid-gas interface.

For superhydrophobic surfaces with air trapped in the grooves and where the working fluid is water the ratio of fluid viscosities is typically $1 / 55 \approx 0.02$, which is significantly less than unity. For liquid-infused surfaces Solomon, Khalil \& Varanasi 
(2014) give a table recording drag reduction characteristics associated with varying working fluid to subphase fluid viscosity ratios and it is noticeable that the viscosity ratios cited are either much smaller, or much larger, than unity. It is therefore natural to contemplate carrying out a formal perturbation analysis of the problem with the viscosity contrast taken as the expansion parameter. Such a perturbation analysis, with a view to gaining theoretical insight into the effect on slip of an enclosed subphase gas, does not appear to have been carried out before. In this paper we carry out such an analysis for the case of a weakly viscous subphase gas; in the supplementary material available at https://doi.org/10.1017/jfm.2017.274, we include details of the generalization of our approach to a highly viscous subphase more relevant to so-called liquid-infused surfaces.

On the other hand, a perturbation approach to incorporating non-zero meniscus curvature has already been explored. Sbragaglia \& Prosperetti (2007) calculated the first-order correction to the effective longitudinal slip length for flows in channels assuming that the curvature of the meniscus is small. Their analysis places no restriction on the no-shear fraction of the surface. Proceeding from a complementary direction, the present author (Crowdy 2010) has studied the same problem of longitudinal shear flow over a periodic array of protruding menisci using a conformal geometric approach that places no restriction on the protrusion angle but which is valid in the dilute limit of small no-shear fraction (hence, the no-shear fraction is now the relevant perturbation expansion parameter). An explicit formula for the effective slip length was found. The author has since performed a higher-order analysis in the no-shear fraction (Crowdy 2016) producing explicit slip length formulas that are now nonlinear in both protrusion angle and no-shear fraction and which give excellent approximations to the effective slip length even at large no-shear fractions, and still without restriction on the protrusion angle. The latter results pertain to semi-infinite shear flows over the surfaces and are not directly relevant to channel flows although they can be used to approximate hydrodynamic slip in large aspect ratio channel flows via asymptotic methods. Schnitzer (2016) has built on these results to find asymptotic formulas valid at even higher no-shear fractions.

We now summarize the main results of the present paper. The flow situation to be considered is longitudinal pressure-driven flow in a channel of height $h$ comprising an upper no-slip wall and a lower wall made up of a periodic array of curved circular-arc menisci spanning rectangular grooves of depth $H$. We also treat the case in which $h \rightarrow$ $\infty$ and contemplate that, instead of a pressure-driven flow, a shear flow, with imposed shear rate $\dot{\gamma}$, exists in the semi-infinite region above the meniscus; this semi-infinite case has been considered theoretically by several other authors using different methods (Nizkaya et al. 2014; Schönecker et al. 2014). The period of the surface is $2 a$ and the meniscus occupies width $2 b$; the protrusion angle is $\theta \ll 1$; thus we assume that the meniscus is only weakly deformed from the flat state. Figure 1 shows a schematic of a typical period window. Region $D$ is occupied by fluid of viscosity $\eta_{1}$ and region $G$ contains fluid of viscosity $\eta_{2}$ where, in the main body of this paper, we assume that the viscosity ratio

$$
\epsilon \equiv \frac{\eta_{2}}{\eta_{1}} \ll 1 .
$$

Under the assumption that the two non-dimensional parameters $\theta \sim \epsilon \ll 1$ are of the same order, our principal result is to show that an integral expression for the effective slip length $\lambda$ associated with the pressure-driven flow is

$$
\lambda=\frac{2}{a h^{2} S^{2}} \int_{\text {meniscus }}\left[-w_{P} \frac{\partial\left(w_{P}+\mathcal{P}\right)}{\partial n}+\epsilon w_{P} \frac{\partial W_{0}}{\partial n}\right] \mathrm{d} s+o(\epsilon, \theta) .
$$




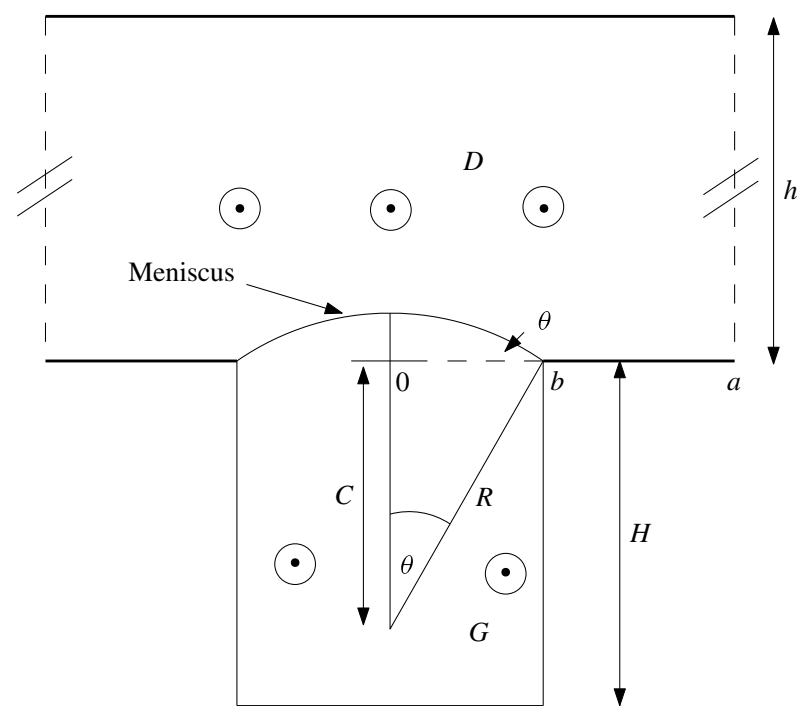

FIGURE 1. Single period window, in an $(x, y)$ plane, for longitudinal flow in a channel of height $h$ with a periodic array of rectangular grooves of height $H$. The upper wall is a no-slip surface. The meniscus protrusion angle $\theta$ is assumed to be small. Domain $D$ is occupied by fluid of viscosity $\eta_{1}$, the groove $G$ by fluid of viscosity $\eta_{2}$ with $\epsilon=\eta_{2} / \eta_{1} \ll 1$.

Here $\partial / \partial n$ denotes the outward normal derivative on the meniscus. In this expression $\mathcal{P}$ denotes a simple Poiseuille flow in a rectangular channel - see (2.15) - and $w_{P}(x, y)$ is Philip's solution for longitudinal flow over a periodic array of flat no-shear slots along the lower wall (Philip 1972); the latter solution has been found in closed form. The function $W_{0}$ is the leading-order flow induced in a rectangular groove by the known flow $w_{P}$ for a flat meniscus; we show here, using complex variable methods, that $W_{0}$ can also be found in analytical form. All these observations imply that the integrand in (1.3) is known. Consequently (1.3) can be used to infer the following formulas for the first-order corrections to the slip length. With the constant $S \equiv-\left(1 / \eta_{1}\right) \partial p / \partial Z$ encoding the longitudinal pressure gradient along the $Z$ direction in an $(x, y, Z)$ plane then in the expansion

$$
\lambda=\lambda_{P}+\theta \lambda^{(\theta)}+\epsilon \Lambda^{(\epsilon)}+o(\epsilon, \theta)
$$

we show here that

$$
\lambda^{(\theta)}=\lambda_{1}^{(\theta)}+\lambda_{2}^{(\theta)}, \quad \Lambda^{(\epsilon)}=-\frac{1}{a \pi h^{2} S^{2}} \sum_{n \geqslant 1} \frac{\mathcal{I}_{2 n-1}^{2}}{(2 n-1)},
$$

where $\lambda_{P}$ is the slip length found by Philip (1972) and

$$
\left.\begin{array}{c}
\lambda_{1}^{(\theta)} \equiv-\frac{1}{a b h^{2} S} \int_{-b}^{b}\left(b^{2}-x^{2}\right)\left[w_{P}(x, 0)-\frac{1}{S}\left(\frac{\partial w_{P}(x, 0)}{\partial x}\right)^{2}\right] \mathrm{d} x, \\
\lambda_{2}^{(\theta)} \equiv-\frac{1}{a b h^{2} S} \int_{-b}^{b}\left(b^{2}-x^{2}\right) w_{P}(x, 0) \mathrm{d} x,
\end{array}\right\}
$$


and

$$
\mathcal{I}_{n}=\int_{-b}^{b} \frac{\partial w_{P}(x, 0)}{\partial x}\left[\frac{1}{\xi(x)^{n}}+\xi(x)^{n}\right] \mathrm{d} x
$$

where

$$
\xi(z) \equiv \frac{P\left(-\mathrm{ie}^{-\mathrm{i} \pi z / 2 b}, \rho\right)-P\left(\mathrm{ie}^{-\mathrm{i} \pi z / 2 b}, \rho\right)}{P\left(-\mathrm{ie}^{-\mathrm{i} \pi z / 2 b}, \rho\right)+P\left(\mathrm{ie}^{-\mathrm{i} \pi z / 2 b}, \rho\right)}, \quad \rho=\mathrm{e}^{-\pi H /(2 b)},
$$

with

$$
P(\eta, \rho) \equiv(1-\eta) \prod_{n=1}^{\infty}\left(1-\rho^{2 n} \eta\right)\left(1-\rho^{2 n} / \eta\right) .
$$

Formulas (1.3)-(1.9) are the main results of the paper.

Ignoring subphase gas effects, Sbragaglia \& Prosperetti (2007) studied the effect of weak meniscus curvature on the slip length in unidirectional channels. They calculate the slip length correction as an expansion

$$
\lambda=\lambda^{(0, h)}+\tilde{\epsilon} \lambda^{(1, h)}+o(\tilde{\epsilon}),
$$

where their non-dimensional expansion parameter $\tilde{\epsilon} \ll 1$ is related to the protrusion angle $\theta$ (and is not to be confused with our viscosity ratio parameter $\epsilon$ ). In the semiinfinite case $h \rightarrow \infty$ Sbragaglia \& Prosperetti (2007) report an integral expression for the first-order slip length correction (rewritten in the notation of figure 1):

$$
\frac{\tilde{\epsilon} \lambda^{(1, \infty)}}{2 \pi}=-\frac{b^{2}}{4 R a} F(\delta), \quad \delta=\frac{b}{a}, \quad F(\delta)=\delta \int_{0}^{1}\left(1-x^{2}\right) \frac{[1-\cos (x \pi \delta)] \mathrm{d} x}{\cos (x \pi \delta)-\cos (\pi \delta)} .(1.11 a-c)
$$

For channel flows with $h<\infty$, Sbragaglia \& Prosperetti (2007) offer no analogous integral formula; instead the first-order slip length correction is found numerically by solving an infinite set of dual series equations coupling expansion coefficients for the first-order velocity correction with the required slip length correction. Formulas (1.5) and (1.6) reveal that solving such an infinite linear system is not necessary and that an integral expression akin to (1.11) exists even for finite channel heights $h<\infty$. This result appears to be new.

The theoretical approach here rests on a combination of perturbation theory with use of a 'reciprocal formula' based on Green's second integral identity. While Sbragaglia \& Prosperetti (2007) did not use any such reciprocity arguments ours is not the first study to exploit the latter to understand superhydrophobic surfaces: Squires (2008) employed reciprocal theorems to examine electrokinetic effects on flat slipping surfaces; Baier, Steffes \& Hardt (2010) used them to study thermal Marangoni flow over a superhydrophobic array of fins oriented parallel or perpendicular to an applied temperature gradient. None of these prior works using reciprocity ideas, however, take non-zero meniscus curvature into account.

\section{Perturbation theory and reciprocity}

The flow is periodic in the $x$-direction so attention is restricted to the single period window shown in figure 1 . We divide the flow into regions $D$ and $G$ where $D$ is occupied by a 'working fluid' of viscosity $\eta_{1}$ and $G$ is the groove region filled with fluid of viscosity $\eta_{2}$. We define the non-dimensional parameter

$$
\epsilon=\frac{\eta_{2}}{\eta_{1}}
$$


and assume it is small, $\epsilon \ll 1$. In Cartesian coordinates $(x, y, Z)$ we assume unidirectional flow in the $Z$ direction with $w$ and $W$ denoting the longitudinal velocities in regions $D$ and $G$ respectively so that the fluid velocity $\boldsymbol{u}$ is

$$
\boldsymbol{u}= \begin{cases}(0,0, w(x, y)), & (x, y) \text { in } D \\ (0,0, W(x, y)), & (x, y) \text { in } G\end{cases}
$$

In the subphase groove there are several choices to be made depending on the experimental set-up. Here we assume that the subphase gas is simply dragged along by the working pressure-driven fluid in region $D$. Other choices are to assume 'dead-end grooves' which will lead to an induced pressure gradient in region $G$; adaptations of the analysis herein to this case can be made. In our case, the governing partial differential equations for $w$ and $W$ are

$$
\nabla^{2} w=-\frac{1}{\eta_{1}} \frac{\partial p}{\partial Z} \equiv-S, \quad \nabla^{2} W=0 .
$$

In region $D$ the boundary condition on the no-slip walls at the top of the channel and on the portions of the plane $y=0$ away from the menisci is

$$
w=0 .
$$

Periodicity conditions are imposed on the side walls of region $D$. The boundary conditions in the groove $G$ are that

$$
W=0
$$

on the no-slip side walls and on the no-slip lower wall at $y=-H$. On the curved meniscus, where the two fluids meet, we have boundary conditions

$$
w=W, \quad \frac{\partial w}{\partial n}=\epsilon \frac{\partial W}{\partial n},
$$

where $\partial / \partial n$ denotes a normal derivative reflecting the requirement that the fluid velocities and shear stresses are continuous at the interface.

Let $w_{P}$ be the solution, with the same driving pressure gradient, for longitudinal flow over a periodic array of flat menisci as found by Philip (1972) and let $\lambda_{P}$ be the effective slip length for such a flow. Within a perturbation approach which assumes that the meniscus deflection from the flat state is small and accounting now for weakly viscous fluid in the subphase we can write the expansions

$$
\left.\begin{array}{c}
w=w_{P}+\theta w_{1}^{(\theta)}+\epsilon w_{1}^{(\epsilon)}+o(\epsilon, \theta), \\
W=W_{0}+\theta W_{1}^{(\theta)}+\epsilon W_{1}^{(\epsilon)}+o(\epsilon, \theta), \\
\lambda=\lambda_{P}+\theta \lambda^{(\theta)}+\epsilon \Lambda^{(\epsilon)}+o(\epsilon, \theta),
\end{array}\right\}
$$

where $w_{P}$ and $\lambda_{P}$ are known, but all other quantities in the expansions (2.7) are unknown. $\lambda$ is the effective slip length incorporating the first-order corrections

$$
\theta \lambda^{(\theta)}+\epsilon \Lambda^{(\epsilon)},
$$

which are the key quantities of interest in this paper. 
On substitution of the expansions (2.7) into the first of the boundary conditions (2.6) we find, at leading order,

$$
W_{0}=w_{P},
$$

which provides a boundary condition on the leading-order flow $W_{0}$ in the subphase region $G$. It shows that, at leading order, the gas in the groove only moves because it is dragged along by the upper working fluid. All other conditions obtained at higher order in $\theta$ and $\epsilon$ from this boundary condition will not be needed in what follows.

Turning to the second stress balance boundary condition in (2.6) at first order in $\theta$ and $\epsilon$ we find

$$
\frac{\partial w_{P}}{\partial n}+\theta \frac{\partial w_{1}^{(\theta)}}{\partial n}=0, \quad \frac{\partial w_{1}^{(\epsilon)}}{\partial n}=\frac{\partial W_{0}}{\partial n},
$$

where we recall that since the meniscus is only weakly deflected then $\partial w_{P} / \partial n$ is $O(\theta)$. These boundary conditions are the only ones needed in what follows.

By Green's second identity,

$$
\iint_{D}\left[w \nabla^{2} w_{P}-w_{P} \nabla^{2} w\right] \mathrm{d} A=\oint_{\partial D}\left[w \frac{\partial w_{P}}{\partial n}-w_{P} \frac{\partial w}{\partial n}\right] \mathrm{d} s,
$$

where $\mathrm{d} s$ denotes an element of arclength and $\partial / \partial n$ denotes the outward normal derivative. $\partial D$ denotes the boundary of $D$. Suppose $Q$ is the volume flux through $D$ associated with the flow $w$ and let $Q_{P}$ be the flux through $D$ of Philip's channel flow solution $w_{P}$. Since $\nabla^{2} w_{p}=\nabla^{2} w=-S$ then (2.11) implies, on substitution of (2.7),

$$
\begin{aligned}
S\left(Q_{P}-Q\right)= & \int_{\text {meniscus }}\left(w_{P}+\theta w_{1}^{(\theta)}+\epsilon w_{1}^{(\epsilon)}\right) \frac{\partial w_{P}}{\partial n} \mathrm{~d} s \\
& -\int_{\text {meniscus }} w_{P} \frac{\partial}{\partial n}\left(w_{P}+\theta w_{1}^{(\theta)}+\epsilon w_{1}^{(\epsilon)}\right) \mathrm{d} s+o(\epsilon, \theta),
\end{aligned}
$$

where, to eliminate all other contributions to the surface integral, we have used the periodicity of $w$ and $w_{P}$ in the $x$ direction, and the no-slip conditions satisfied by both flows on the solid wall portions of $\partial D$. But, after a cancellation of terms, and recalling the fact that $\partial w_{P} / \partial n$ is $O(\theta)$, then

$$
S\left(Q_{P}-Q\right)=-\int_{\text {meniscus }}\left[w_{P} \theta \frac{\partial w_{1}^{(\theta)}}{\partial n} \mathrm{~d} s+w_{P} \epsilon \frac{\partial w_{1}^{(\epsilon)}}{\partial n}\right] \mathrm{d} s+o(\epsilon, \theta) .
$$

On use of the boundary conditions (2.10), we find

$$
S\left(Q_{P}-Q\right)=\int_{\text {meniscus }}\left[w_{P} \frac{\partial w_{P}}{\partial n} \mathrm{~d} s-w_{P} \epsilon \frac{\partial W_{0}}{\partial n}\right] \mathrm{d} s+o(\epsilon, \theta) .
$$

To determine $Q_{P}$ a second invocation of the reciprocal theorem is useful. Let $\mathcal{P}$ denote the Poiseuille flow in the channel, that is,

$$
\mathcal{P}=\frac{S y}{2}(h-y) .
$$

Now Green's identity implies that

$$
\iint_{D}\left[w_{P} \nabla^{2} \mathcal{P}-\mathcal{P} \nabla^{2} w_{P}\right] \mathrm{d} A=\oint_{\partial D}\left[w_{P} \frac{\partial \mathcal{P}}{\partial n}-\mathcal{P} \frac{\partial w_{P}}{\partial n}\right] \mathrm{d} s .
$$


But, if $Q_{\mathcal{P}}$ is the volume flux through $D$ of the Poiseuille flow, then (2.16) leads to

$$
S\left(Q_{P}-Q_{\mathcal{P}}\right)=-\int_{\text {meniscus }}\left[w_{P} \frac{\partial \mathcal{P}}{\partial n}-\mathcal{P} \frac{\partial w_{P}}{\partial n}\right] \mathrm{d} s=-\int_{\text {meniscus }} w_{P} \frac{\partial \mathcal{P}}{\partial n} \mathrm{~d} s+o(\theta),
$$

where we have used the $x$-periodicity of $\mathcal{P}$ and $w_{P}$ and the no-slip conditions on the solid wall portions of $\partial D$ satisfied by both flows. We have also used the fact that the quantity

$$
\int_{\text {meniscus }} \mathcal{P} \frac{\partial w_{P}}{\partial n} \mathrm{~d} s=o(\theta)
$$

since both $\mathcal{P}$ and $\partial w_{P} / \partial n$ vanish on $y=0$ so each will be $O(\theta)$ on the weakly perturbed interface. We can now eliminate $Q_{P}$ between (2.14) and (2.17) to find

$$
Q=Q_{\mathcal{P}}+\frac{1}{S} \int_{\text {meniscus }}\left[-w_{P} \frac{\partial\left(w_{P}+\mathcal{P}\right)}{\partial n}+\epsilon w_{P} \frac{\partial W_{0}}{\partial n}\right] \mathrm{d} s+o(\epsilon, \theta) .
$$

By direct calculation,

$$
\begin{aligned}
\frac{Q_{\mathcal{P}}}{2 S} & =\int_{0}^{b} \mathrm{~d} x \int_{\theta \eta(x)}^{h} \frac{y(h-y)}{2} \mathrm{~d} y+\int_{b}^{a} \mathrm{~d} x \int_{0}^{h} \frac{y(h-y)}{2} \mathrm{~d} y \\
& =\int_{0}^{b}\left[\frac{h y^{2}}{4}-\frac{y^{3}}{6}\right]_{\theta \eta(x)}^{h} \mathrm{~d} x+\int_{b}^{a}\left[\frac{h y^{2}}{4}-\frac{y^{3}}{6}\right]_{0}^{h} \mathrm{~d} x \\
& =\int_{0}^{a}\left[\frac{h y^{2}}{4}-\frac{y^{3}}{6}\right]_{0}^{h} \mathrm{~d} x+o(\theta),
\end{aligned}
$$

where

$$
\eta(x)=\frac{\left(b^{2}-x^{2}\right)}{2 b}
$$

is the parabolic shape of the meniscus (see supplementary material for more details). The consequence is that

$$
Q_{\mathcal{P}}=\frac{a h^{3} S}{6}+o(\theta)
$$

and, on substitution into (2.19),

$$
Q=\left(\frac{a h^{3} S}{6}\right)+\frac{1}{S} \int_{\text {meniscus }}\left[-w_{P} \frac{\partial\left(w_{P}+\mathcal{P}\right)}{\partial n}+\epsilon w_{P} \frac{\partial W_{0}}{\partial n}\right] \mathrm{d} s+o(\epsilon, \theta) .
$$

We now compare (2.23) with the relation for the flux $Q_{\text {eff }}$ through a channel with the same driving pressure gradient but now with a flat lower wall on which a Navier-slip condition with slip parameter $\lambda$ is imposed. We will follow Sbragaglia \& Prosperetti (2007) and use the formula

$$
Q_{\text {eff }}=\frac{a h^{3} S}{6}\left(1+\frac{3 \lambda}{h}\right)
$$

even though this is really a linear approximation for $\lambda / h \ll 1$ of the actual result

$$
Q_{\text {eff }}=\frac{a h^{3} S}{6}\left(\frac{1+4 \lambda / h}{1+\lambda / h}\right)
$$


obtained by solving the channel flow problem with a Navier-slip condition $\lambda \mathrm{d} w / \mathrm{d} y=$ $w$ imposed on the lower boundary $y=0$. A comparison of (2.23) and (2.24) implies

$$
\frac{a h^{2} \lambda S}{2}=\frac{1}{S} \int_{\text {meniscus }}\left[-w_{P} \frac{\partial\left(w_{P}+\mathcal{P}\right)}{\partial n}+\epsilon w_{P} \frac{\partial W_{0}}{\partial n}\right] \mathrm{d} s+o(\epsilon, \theta),
$$

which leads to our key result (1.3), and which involves only the leading-order solutions $w_{P}$ and $W_{0}$. It is already clear from (2.26) that, to first order in $\theta$ and $\epsilon$, it is unnecessary to compute either of the first-order flow corrections in regions $D$ and $G$ and that only the leading-order flows in each subdomain are needed to compute the first-order slip length modification. Recall that the solution $w_{P}$ has been found in closed form by Philip (1972). The solution $W_{0}$ for the leading-order flow in region $G$ is itself uniquely determined by $w_{P}$ through the boundary condition (2.9) which has not yet been used in the analysis. In $\S 4$ we show how to solve the boundary value problem for $W_{0}$ using complex variable methods.

Finally, in the limit $h \rightarrow \infty$, so that we consider instead a flow in region $D$ tending to a semi-infinite linear shear with

$$
w \rightarrow \dot{\gamma}(y+\lambda), \quad \text { as } y \rightarrow \infty,
$$

the result (2.26) must be modified. The analysis starts again from formula (2.11) but now, since there is no driving pressure gradient, the left-hand side area integral vanishes identically. However the surface integral on the right-hand side acquires a new non-zero contribution from $y \rightarrow \infty$ yielding

$$
0=\int_{\text {meniscus }}\left[w_{P} \frac{\partial w_{P}}{\partial n}-\epsilon w_{P} \frac{\partial W_{0}}{\partial n}\right] \mathrm{d} s+2 a \dot{\gamma}^{2}\left(\lambda-\lambda_{P}\right)+o(\epsilon, \theta),
$$

or, on rearrangement,

$$
\lambda=\lambda_{P}+\frac{1}{2 a \dot{\gamma}^{2}} \int_{\text {meniscus }}\left[-w_{P} \frac{\partial w_{P}}{\partial n}+\epsilon w_{P} \frac{\partial W_{0}}{\partial n}\right] \mathrm{d} s+o(\epsilon, \theta) .
$$

\section{Meniscus curvature effects: $\epsilon=0, \theta \neq 0$}

First we first ignore subphase gas effects and set $\epsilon=0$. Sbragaglia \& Prosperetti (2007) used an approach based on dual series equations and find it necessary to solve for the first-order flow correction in region $D$ in order to deduce the first-order slip length correction. We know now that formula (2.26) offers a more direct route to the same result.

We write Philip's channel flow solution (Philip 1972) in the form

$$
w_{P}=-\frac{S y^{2}}{2}+\hat{w},
$$

where the function $\hat{w}$ is harmonic. In the supplementary materials full details are given of how, starting from (2.26), one can derive the formulas

$$
\frac{a h^{2} S \lambda_{P}}{2}=\int_{-b}^{b} \frac{h}{2} \hat{w}(x, 0) \mathrm{d} x, \quad \text { or } \quad \lambda_{P}=\frac{1}{a h S} \int_{-b}^{b} \hat{w}(x, 0) \mathrm{d} x,
$$


and

$$
\lambda^{(\theta)}=-\frac{4}{a h^{2} S} \int_{-b}^{b} \eta(x)\left[\hat{w}(x, 0)-\frac{1}{2 S}\left(\frac{\partial \hat{w}(x, 0)}{\partial x}\right)^{2}\right] \mathrm{d} x=\lambda_{1}^{(\theta)}+\lambda_{2}^{(\theta)},
$$

where we separate the final result into the two contributions

$$
\left.\begin{array}{c}
\lambda_{1}^{(\theta)} \equiv-\frac{2}{a h^{2} S} \int_{-b}^{b} \eta(x)\left[\hat{w}(x, 0)-\frac{1}{S}\left(\frac{\partial \hat{w}(x, 0)}{\partial x}\right)^{2}\right] \mathrm{d} x, \\
\lambda_{2}^{(\theta)} \equiv-\frac{2}{a h^{2} S} \int_{-b}^{b} \eta(x) \hat{w}(x, 0) \mathrm{d} x .
\end{array}\right\}
$$

The quantity $\partial \hat{w}(x, 0) / \partial x$ has inverse square root singularities at the integration end points $x= \pm b$ but the integrand in the expression for $\lambda^{(\theta)}$ is nevertheless regular since $\eta(x)$ has simple zeros at those points. From (3.1) we notice that, on $y=0$, $\hat{w}(x, 0)=w_{P}(x, 0)$ and $\partial \hat{w}(x, 0) / \partial x=\partial w_{P}(x, 0) / \partial x$ which leads, after substitution of the expression (2.21) for $\eta(x)$, to formulas (1.6).

Sbragaglia \& Prosperetti (2007) state their (non-dimensionalized) slip length in the form

$$
\lambda^{(L)}=\lambda^{(0, L)}+\tilde{\epsilon}\left[\lambda_{1}^{(1, L)}+\lambda_{2}^{(1, L)}\right]+o(\tilde{\epsilon}),
$$

where the leading-order slip length is also decomposed into two parts. In their paper all lengths are non-dimensionalized with respect to $a^{*} / \pi$; the non-dimensional expansion parameter $\tilde{\epsilon}$ is related to angle $\theta$ via

$$
\tilde{\epsilon}^{*}=\frac{1}{2 R^{*}}=-\frac{\theta}{2 b^{*}}=\frac{\pi}{a^{*}} \tilde{\epsilon},
$$

where we have added asterisks to emphasize dimensional quantities. We have also added a minus sign in front of angle $\theta$ because a depressed meniscus (rather than a protruding one) was studied in Sbragaglia \& Prosperetti (2007). It follows that the nondimensional expansion parameters used here and in Sbragaglia \& Prosperetti (2007) are related by

$$
\tilde{\epsilon}=-\frac{a^{*} \theta}{2 \pi b^{*}} .
$$

The contribution $\lambda_{2}^{(1)}$ found here precisely corresponds to the quantity $\lambda_{2}^{(1, L)}$ found by Sbragaglia \& Prosperetti (2007) after appropriate non-dimensionalization. Indeed, it can be verified that

$$
\tilde{\epsilon} \lambda_{2}^{(1, L)}=-\theta\left(\frac{\pi}{a^{*}}\right) \lambda_{2}^{(\theta) *},
$$

where, on the right-hand side, we have non-dimensionalized our result $\lambda_{2}^{(\theta) *}$ in the same way adopted in Sbragaglia \& Prosperetti (2007). To check (3.8), on substituting from (3.7), it becomes

$$
\frac{a^{*} \theta}{2 \pi b^{*}} \lambda_{2}^{(1, L)}=-\theta\left(\frac{\pi}{a^{*}}\right) \lambda_{2}^{(\theta) *}
$$

implying that

$$
\lambda_{2}^{(1, L)}=\frac{\pi^{2}}{a^{* 2}}\left(2 b^{*} \lambda_{2}^{(\theta) *}\right)=\frac{\pi^{2}}{a^{* 2}}\left[\frac{2}{a^{*} h^{* 2} S}\right] \int_{-b^{*}}^{b^{*}}\left(b^{* 2}-x^{* 2}\right) w_{P}^{*}(x, 0) \mathrm{d} x^{*} .
$$




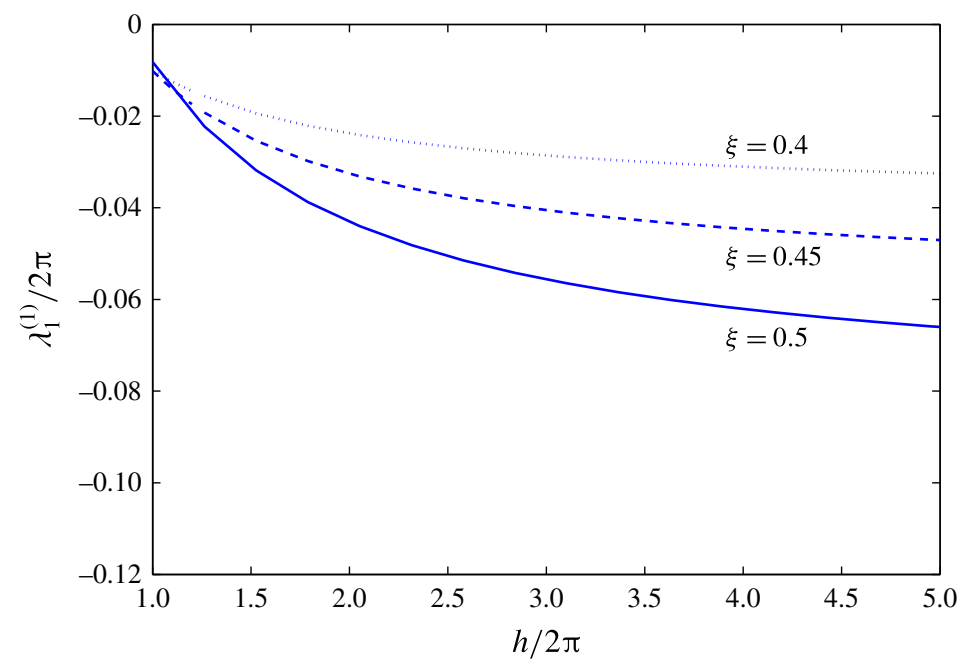

FIGURE 2. (Colour online) Normalized slip length $\lambda_{1}^{(1)} / 2 \pi$ (as a function of channel height) computed using the new integral formula (1.6). This graph should be compared with that in figure 4 of Sbragaglia \& Prosperetti (2007).

With the non-dimensionalizations used in Sbragaglia \& Prosperetti (2007),

$$
b^{*}=\frac{a^{*}}{\pi} b, \quad h^{*}=\frac{a^{*}}{\pi} h, \quad x^{*}=\frac{a^{*}}{\pi} x, \quad w_{P}^{*}(x, 0)=S\left(\frac{a^{*}}{\pi}\right)^{2} \tilde{w}_{P}(x, 0), \quad(3.11 a-d)
$$

and (3.10) reduces to

$$
\lambda_{2}^{(1, L)}=\frac{2}{\pi h^{2}} \int_{-b}^{b}\left(b^{2}-x^{2}\right) \tilde{w}_{P}(x, 0) \mathrm{d} x,
$$

which is precisely formula (42) of Sbragaglia \& Prosperetti (2007) (once we rename $h$ as $L$ and $b$ as $c$ ).

From this point of view, a new result here is to show that the quantity $\lambda_{1}^{(1, L)}$ considered in Sbragaglia \& Prosperetti (2007) is in fact given by the integral formula

$$
\lambda_{1}^{(1, L)}=\frac{2}{\pi h^{2}} \int_{-b}^{b}\left(b^{2}-x^{2}\right)\left[\tilde{w}_{P}(x, 0)-\left(\frac{\partial \tilde{w}_{P}(x, 0)}{\partial x}\right)^{2}\right] \mathrm{d} x,
$$

where, in the original work, it was computed from the numerical solution of a truncated set of dual series equations.

Figure 2 shows a graph of the normalized slip length correction $\lambda_{1}^{(1)} / 2 \pi$ as a function of normalized channel height calculated from the new integral formula (3.13). It should be compared with figure 4 of Sbragaglia \& Prosperetti (2007) where the same graph was plotted based on the numerical solution of dual series equations.

In the supplementary material we rederive (1.11) relevant to the case of semi-infinite shear flow $h \rightarrow \infty$ in an analogous way that differs from the dual series methods of Sbragaglia \& Prosperetti (2007). We show that, in this case, equation (2.29) with $\epsilon=0$ leads to

$$
\lambda=\lambda_{P}+\theta \lambda^{(\theta)}+\cdots, \quad \lambda^{(\theta)}=\frac{\theta}{2 a} \int_{-b}^{b} \eta\left(\frac{\partial w_{P}}{\partial x}\right)^{2} \mathrm{~d} x
$$

and this formula is equivalent to (1.11). 

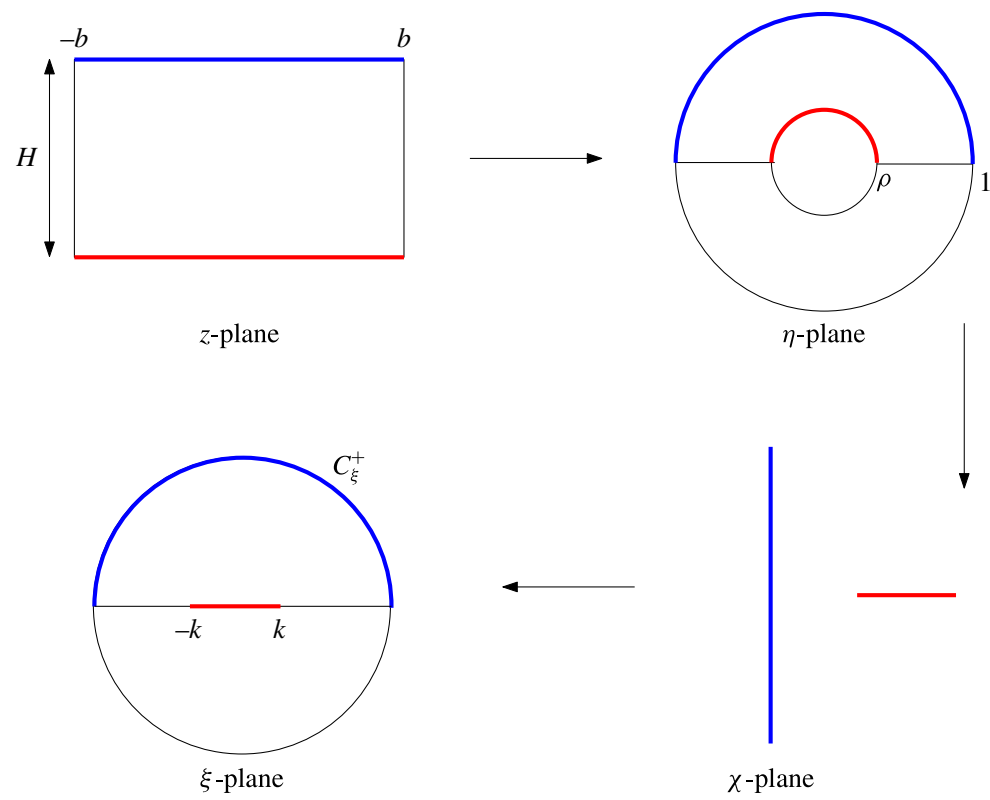

FIGURE 3. (Colour online) Sequence of conformal mappings to construct the mapping between the groove region to the upper half-unit disc in a complex $\xi$-plane. The top edge of the rectangular groove, which neighbours with the working fluid, is transplanted to $C_{\xi}^{+}$; the other three sides are transplanted to the real diameter $[-1,1]$.

\section{Subphase fluid effects: $\epsilon \neq 0, \theta=0$}

The boundary value problem for the leading-order flow $W_{0}$ in the subphase fluid is a Dirichlet problem

$$
\nabla^{2} W_{0}=0
$$

with boundary conditions

$$
W_{0}=w_{P}
$$

on the top of the rectangular groove $y=0,-b<x<b$ and

$$
W_{0}=0
$$

on its other three no-slip walls. The nature of the flow in the upper fluid is communicated to the subphase gas via the boundary condition (4.2).

\subsection{Conformal mapping}

To solve for $W_{0}$ it is useful to construct the conformal mapping from the rectangular groove to an upper half-unit disc, in a complex $\xi$-plane with the upper half-circle denoted by $C_{\xi}^{+}$corresponding to the flat meniscus (i.e. the upper edge of the rectangle). This is done by composing a sequence of simpler transformations illustrated in figure 3. The logarithmic mapping

$$
z \mapsto \frac{2 \mathrm{i} b}{\pi} \log \eta+b
$$


transplants the upper half-annulus in a complex $\eta$-plane

$$
\operatorname{Im}[\eta]>0, \quad \rho<|\eta|<1,
$$

with

$$
H=-\frac{2 b}{\pi} \log \rho, \quad \text { or } \quad \rho=\mathrm{e}^{-\pi H /(2 b)}
$$

to the rectangular groove. Relation (4.4) implies that

$$
\eta=\mathrm{ie}^{-\mathrm{i} \pi z / 2 b} \text {. }
$$

Next let

$$
\eta \mapsto \chi=\frac{P(\eta, \rho)}{P(-\eta, \rho)},
$$

where the special function $P(\eta, \rho)$ is defined in (1.9). The latter function can easily be shown, directly from its infinite product definition (1.9), to satisfy:

$$
P\left(\rho^{2} \eta, \rho\right)=-\frac{P(\eta, \rho)}{\eta}, \quad P(1 / \eta, \rho)=-\frac{P(\eta, \rho)}{\eta} .
$$

These functional relations can be used to show that the function (4.8) transplants the entire annulus $\rho<|\eta|<1$ to the right half-plane with the circle $|\eta|=\rho$ mapping to a slit along the positive real $\eta$-axis. Finally, if we now apply the Cayley mapping

$$
\chi \mapsto \xi=\frac{1-\chi}{1+\chi}
$$

then the right half- $\chi$-plane containing the excised slit is transplanted to the interior of the unit $\zeta$-circle with an excised slit along its real diameter. The composition is the mapping (1.8) reported earlier. If we view $z=z(\xi)=x(\xi, \bar{\xi})+\mathrm{i} y(\xi, \bar{\xi})$ as a function of $\xi$ then the properties (4.9) can be used to demonstrate that, for $|\xi|=1$,

$$
x(\bar{\xi}, \xi)=x(\xi, \bar{\xi})
$$

which will be useful later.

\subsection{Solution of boundary value problem}

Let us define the complex potential $\mathcal{H}_{0}(\xi) \equiv h_{0}(z)$ such that

$$
W_{0}=\operatorname{Im}\left[\mathcal{H}_{0}(\xi)\right] \text {. }
$$

By the no-slip conditions on the three submerged edges of the groove we know that on the real diameter $\xi \in[-1,1]$

$$
\operatorname{Im}\left[\mathcal{H}_{0}(\xi)\right]=0
$$

so that

$$
\overline{\mathcal{H}}_{0}(\xi)=\mathcal{H}_{0}(\xi),
$$

where the so-called Schwarz conjugate of an analytic function $f(z)$ is defined by $\bar{f}(z) \equiv$ $\overline{f(\bar{z})}$. By the Schwarz reflection principle this relation allows us both to analytically 
continue the complex potential into the lower half- $\xi$-plane and to deduce information on the imaginary part of the potential on the lower half-unit $\xi$-circle, denoted by $C_{\xi}^{-}$, given these data on the upper half-unit circle. Specifically, if $\xi \in C_{\xi}^{-}$then $\bar{\xi} \in C_{\xi}^{+}$and we have that

$$
\operatorname{Re}\left[-\mathrm{i} \mathcal{H}_{0}(\xi)\right]=\operatorname{Re}\left[+\mathrm{i} \overline{\mathcal{H}_{0}}(\bar{\xi})\right]=\operatorname{Re}\left[+\mathrm{i} \mathcal{H}_{0}(\bar{\xi})\right]=-\operatorname{Re}\left[-\mathrm{i} \mathcal{H}_{0}(\bar{\xi})\right] .
$$

Hence, by (4.2), we have

$$
\operatorname{Re}\left[-\mathrm{i} \mathcal{H}_{0}(\xi)\right]= \begin{cases}w_{P}(x(\xi, \bar{\xi}), 0), & \xi \in C_{\xi}^{+}, \\ -w_{P}(x(\bar{\xi}, \xi), 0), & \xi \in C_{\xi}^{-} .\end{cases}
$$

The sought-after function $-\mathrm{i} \mathcal{H}_{0}(\xi)$, by the Schwarz reflection principle, is analytic everywhere in the unit $\xi$-disc. Moreover, from (4.16), its real part is known everywhere on the boundary of the unit disc. The boundary value problem for $-i \mathcal{H}_{0}(\xi)$ is of the standard Schwarz type. The solution can be written down explicitly in one of two ways: either using the classical Poisson integral formula (which leads to a singular integral representation for $-\mathrm{i} \mathcal{H}_{0}(\xi)$ ), or by finding the coefficients of a Taylor series. We adopt the latter method.

Define the Laurent series

$$
\sum_{n=-\infty}^{\infty} p_{n} \xi^{n}= \begin{cases}w_{P}(x(\xi, \bar{\xi}), 0), & \xi \in C_{\xi}^{+}, \\ -w_{P}(x(\bar{\xi}, \xi), 0), & \xi \in C_{\xi}^{-},\end{cases}
$$

representing the given data on $|\xi|=1$. Since the data are real, then $p_{-n}=\overline{p_{n}}, n \geqslant 1$. Using orthogonality these coefficients are given, for $n \geqslant 1$, by

$$
p_{n}=I_{n}+J_{n} \quad n \geqslant 1,
$$

with

$$
I_{n}=\frac{1}{2 \pi \mathrm{i}} \int_{C_{\xi}^{+}} \frac{w_{P}(x(\xi, \bar{\xi}), 0)}{\xi^{n+1}} \mathrm{~d} \xi, \quad J_{n}=\frac{1}{2 \pi \mathrm{i}} \int_{C_{\xi}^{-}}-\frac{w_{P}(x(\bar{\xi}, \xi), 0)}{\xi^{n+1}} \mathrm{~d} \xi
$$

On taking a complex conjugate of $J_{n}$ we find

$$
\overline{J_{n}}=\frac{1}{2 \pi \mathrm{i}} \int_{C_{\xi}^{+}}-\frac{\overline{w_{P}}(\bar{x}(\xi, \bar{\xi}), 0)}{\bar{\xi}^{n+1}} \mathrm{~d} \bar{\xi}=-\frac{1}{2 \pi \mathrm{i}} \int_{C_{\xi}^{+}} \frac{w_{P}(x(\bar{\xi}, \xi), 0)}{\bar{\xi}^{n+1}} \mathrm{~d} \bar{\xi}=-I_{n},
$$

where we have used the fact that $w_{P}$ and $x$ are real functions together with property (4.11). Hence

$$
p_{n}=\left[I_{n}-\overline{I_{n}}\right]=2 \mathrm{i} \operatorname{Im}\left[I_{n}\right], \quad n \geqslant 1 .
$$

It is useful to note that

$$
I_{n}=-\frac{1}{2 \pi \mathrm{i} n} \int_{C_{\xi}^{+}} w_{P}(x(\xi, \bar{\xi}), 0) \mathrm{d}\left[\frac{1}{\xi^{n}}\right]=-\frac{1}{2 \pi \mathrm{i} n} \int_{-b}^{b} \frac{\partial w_{P}}{\partial x}(x, 0) \frac{\mathrm{d} x}{\xi(x)^{n}},
$$

where we have used integration by parts, and the fact that $w_{P}$ vanishes at $\xi= \pm 1$, and where a minus sign has been added to account for the fact that traversing $C_{\xi}^{+}$in an 
Subphase gas and meniscus curvature effects for superhydrophobic surfaces

anticlockwise direction implies traversing the meniscus in the $z$-plane from $+b$ to $-b$. Also, on taking a complex conjugate,

$$
\overline{I_{n}}=\frac{1}{2 \pi \mathrm{i} n} \int_{-b}^{b} \frac{\partial w_{P}}{\partial x}(x, 0) \xi(x)^{n} \mathrm{~d} x,
$$

where we have used the fact that $\overline{\xi(x)}=1 / \xi(x)$ on $x \in[-b, b], y=0$.

Now introduce the Taylor expansion, convergent in the unit $\xi$-disc:

$$
-\mathrm{i} \mathcal{H}_{0}(\xi)=a_{0}+\sum_{n=1}^{\infty} a_{n} \xi^{n}
$$

On substitution into the boundary value problem (4.16) for $\mathcal{H}_{0}(\xi)$ and after equating coefficients of powers of $\xi$ we find

$$
\operatorname{Re}\left[a_{0}\right]=0, \quad a_{n}=2 p_{n}, \quad n \geqslant 1 .
$$

Hence,

$$
\mathcal{H}_{0}(\xi)=-4 \sum_{n \geqslant 1} \operatorname{Im}\left[I_{n}\right] \xi^{n}+c
$$

where $c$ is a real constant whose value turns out to be unimportant.

\subsection{Calculation of the slip length correction}

The $O(\epsilon)$ terms in both (1.3) (pertaining to pressure-driven channel flow) and (2.29) (pertaining to semi-infinite shear flow) depend on the quantity

$$
\int_{\text {meniscus }} w_{P} \frac{\partial W_{0}}{\partial n} \mathrm{~d} s \approx-\int_{-b}^{b} w_{P} \frac{\partial W_{0}}{\partial y} \mathrm{~d} x+O(\theta),
$$

where we have used the fact that on the weakly deformed meniscus $\partial / \partial n=-\partial / \partial y+$ $O(\theta)$ and $\mathrm{d} s \approx \mathrm{d} x+O(\theta)$. By the analyticity of $h_{0}(z)$,

$$
\frac{\partial W_{0}}{\partial y}=\operatorname{Re}\left[\frac{\mathrm{d} h_{0}}{\mathrm{~d} z}\right] .
$$

Hence,

$$
\int_{-b}^{b} w_{P}(x, 0) \frac{\partial W_{0}}{\partial y} \mathrm{~d} x=\operatorname{Re}\left[\int_{-b}^{b} w_{P}(x, 0) \mathrm{d} h_{0}\right]=-\operatorname{Re}\left[\int_{-b}^{b} \frac{\partial w_{P}}{\partial x}(x, 0) \mathcal{H}_{0}(\xi) \mathrm{d} x\right],
$$

where we have used integration by parts and the fact that $w_{P}$ vanishes at $x= \pm b, y=0$. If we now substitute for $\mathcal{H}_{0}$ from (4.26), we find

$$
\int_{-b}^{b} w_{P}(x, 0) \frac{\partial W_{0}}{\partial y} \mathrm{~d} x=4 \sum_{n \geqslant 1} \operatorname{Im}\left[I_{n}\right] \operatorname{Re}\left[\int_{-b}^{b} \frac{\partial w_{P}}{\partial x}(x, 0) \xi(x)^{n} \mathrm{~d} x\right],
$$

where a contribution proportional to the unknown constant $c$ vanishes owing to the oddness of $\partial w_{P} / \partial x$. But

$$
\operatorname{Re}\left[\int_{-b}^{b} \frac{\partial w_{P}}{\partial x}(x, 0) \xi(x)^{n} \mathrm{~d} x\right]=\operatorname{Re}\left[\int_{-b}^{b} \frac{\partial w_{P}}{\partial x}(x, 0) \frac{\mathrm{d} x}{\xi(x)^{n}}\right]=\operatorname{Re}\left[-2 \pi \mathrm{i} n I_{n}\right]=2 \pi n \operatorname{Im}\left[I_{n}\right],
$$


where we have used (4.22) and the fact that $\overline{\xi(x)}=1 / \xi(x)$ when $x \in[-b, b]$ and $y=0$. Relation (4.30) then becomes

$$
\int_{-b}^{b} w_{P}(x, 0) \frac{\partial W_{0}}{\partial y} \mathrm{~d} x=8 \pi \sum_{n \geqslant 1} n\left\{\operatorname{Im}\left[I_{n}\right]\right\}^{2} .
$$

Finally, it is easy to show

$$
\operatorname{Im}\left[I_{n}\right]=\frac{\mathcal{I}_{n}}{4 \pi n},
$$

where $\mathcal{I}_{n}$ is defined in (1.7). The conclusion is that

$$
\int_{\text {meniscus }} w_{P} \frac{\partial W_{0}}{\partial n} \mathrm{~d} s=-\frac{1}{2 \pi} \sum_{n \geqslant 1} \frac{\mathcal{I}_{n}^{2}}{n}+O(\theta),
$$

which leads to the expression for $\Lambda^{(\epsilon)}$ given in (1.5) once account is taken of the fact that the terms for $n$ even turn out to be identically zero. For semi-infinite flow the corresponding $O(\epsilon)$ contribution to the slip length correction, found on substituting (4.34) into (2.29), is

$$
-\frac{\epsilon}{4 \pi a \dot{\gamma}^{2}} \sum_{n \geqslant 1} \frac{\mathcal{I}_{n}^{2}}{n},
$$

where, again, contributions from even integers $n$ vanish.

\section{Discussion}

Philip (1972) determined analytical expressions for both the velocity field, and the effective slip lengths, for semi-infinite shear flow and pressure-driven channel flow over a periodic array of flat no-shear slots on a lower wall. This paper has determined the first-order slip length correction, incorporating both weak meniscus curvature and the additional dissipation of a weakly viscous subphase fluid, to be

$$
\theta\left(\lambda_{1}^{(\theta)}+\lambda_{2}^{(\theta)}\right)+\frac{\eta_{2}}{\eta_{1}} \Lambda^{(\epsilon)},
$$

with explicit formulas determined for all terms, and depending only on Philip's analytical solutions for the leading-order flow in the upper working fluid.

As is clear from its representation in (1.5) the sign of the last term in (5.1) is evidently negative. This is natural since we expect that additional dissipation associated with the working fluid having to drag the viscous subphase along will lead to a decrease in the effective slip for a given pressure gradient. If $\theta<0$, so that the meniscus bows into the groove (which is to be expected if the hydrodynamic pressure in the upper working fluid is large) then the term $\theta \lambda_{2}^{(\theta)}$ is positive - as is also evident from the expression (1.6) - since it is associated with the increase in cross-sectional area of the longitudinal flow. The term $\theta \lambda_{1}^{(\theta)}$ is associated with the change in the axial velocity distribution caused by the deflection of the meniscus and, although it is no longer obvious from (1.6), this term tends to decrease the slip. An explanation for this is that, when the interface bows into the groove, the condition of free shear is moved below the level $y=0$ implying a residual non-zero shear stress there. 


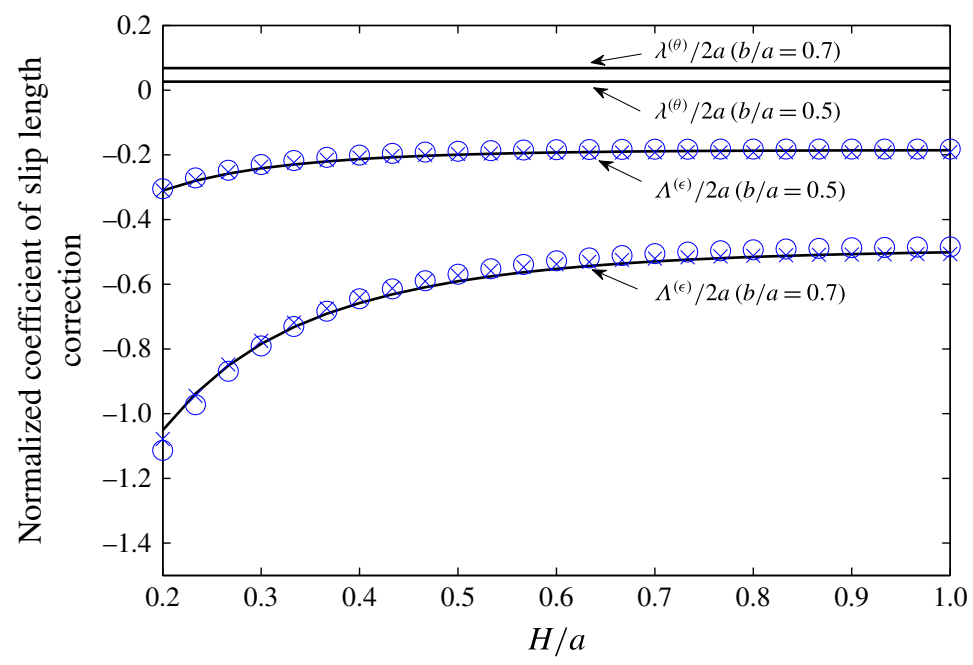

FIGURE 4. (Colour online) Normalized coefficients of the slip length correction for semi-infinite shear flow as a function of groove depth (solid line). Results from formulas derived from the models of Schönecker et al. (2014) (circles) and Nizkaya et al. (2014) (crosses) are also shown.

Even for channels with rectangular grooves, the geometrical parameter space comprising $a, b, h$ and $H$ is large. It is therefore convenient to have available explicit integral formulas in terms of these parameters for the slip length corrections when both weak meniscus curvature and the effect of a weakly viscous subphase gas are incorporated. To gain some insights into the relative importance of the two effects, we focus on the case where the working fluid is a semi-infinite shear flow over the superhydrophobic surface so that $h \rightarrow \infty$. In this case, for $x \in[-b, b]$ and $y=0$ we have (Philip 1972)

$w_{P}(x, 0)=\frac{2 a}{\pi} \cosh ^{-1}\left[\frac{\cos (\pi x / 2 a)}{\cos (\pi b / 2 a)}\right], \quad \frac{\partial w_{P}}{\partial x}(x, 0)=-\frac{\sin (\pi x / 2 a)}{\left[\cos ^{2}(\pi x / 2 a)-\cos ^{2}(\pi b / 2 a)\right]^{1 / 2}}$.

The associated slip length is

$$
\lambda_{P}=\frac{2 a}{\pi} \log \sec \left(\frac{\pi b}{2 a}\right) .
$$

These formulas can simply be substituted into (3.14) and (4.35) to determine the slip length corrections $\lambda^{(\theta)}$ and $\Lambda^{(\epsilon)}$ for this case.

Figure 4 shows a graph of the normalized coefficients $\lambda^{(\theta)} / 2 a$ and $\Lambda^{(\epsilon)} / 2 a$ as a function of normalized groove depth for $b / a=0.5$ and $b / a=0.7$. The quantity $\lambda^{(\theta)} / 2 a$ is independent of groove height, but depends on no-shear fraction $b / a$ and increases in magnitude as the no-shear fraction increases; if $\theta<0$, so that the meniscus bows into the groove, then the net effect is to decrease the slip length. On the other hand, the value of $\Lambda^{(\epsilon)} / 2 a$, which is also negative (so that it always decreases the slip length), depends sensitively on the no-shear fraction and groove depth. Figure 5 shows a graph of the normalized coefficients $\lambda^{(\theta)} / 2 a$ and $\Lambda^{(\epsilon)} / 2 a$ as a function of no-shear fraction $b / a$ for the normalized groove depths $H / a=0.25$ and 1 . For a given no-shear fraction, 


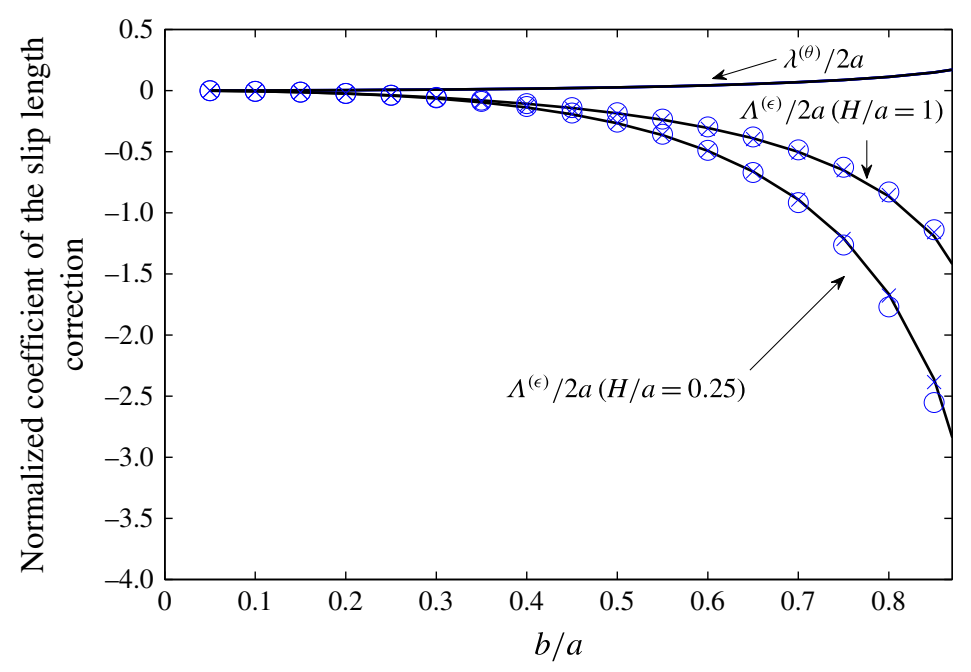

FIgURE 5. (Colour online) Normalized coefficients of the slip length correction for semiinfinite shear flow as a function of no-shear fraction. Results from formulas derived from the models of Schönecker et al. (2014) (circles) and Nizkaya et al. (2014) (crosses) are also shown.

shallower grooves decrease the effective slip more severely than deeper ones as might be expected given that the lower no-slip wall is moving closer to the interface trapping smaller volumes of viscous fluid in the groove so that this trapped fluid is expected to become harder to drag along. For small $H / a$, of order $\theta$, and for $\theta<0$ there will be a lower limit on admissible values of $H / a$ since there will be a critical geometry in which the meniscus touches the lower wall.

Also shown in both figures 4 and 5 are the results obtained from formulas for the effective slip length derived from the models of Nizkaya et al. (2014) and Schönecker et al. (2014); after expanding their formulas for small viscosity ratio $\epsilon$ one can read off expressions for the first-order correction for small $\epsilon$ as predicted by those models. Both are found to give good agreement, for a wide range of parameters, with the results of the perturbation analysis. These models give approximations to the effective slip length for any viscosity ratio; the new formulas of the present paper, while valid only for small viscosity ratios $\epsilon \ll 1$, also give expressions for the effective slip length for pressure-driven flow in channels of finite height and, to the best of our knowledge, no other expressions for the $h<\infty$ case have previously appeared in the literature. Furthermore, in the supplementary materials, we give details of the perturbation analysis in the opposite limit $\epsilon \gg 1$ (and also compare the results derived from the models of Nizkaya et al. (2014) and Schönecker et al. (2014) in that case). Since, as mentioned in the Introduction, in many practical settings the viscosity contrast is either small or large, we expect the new formulas of this paper to be useful additions to the literature. Moreover, it is important to emphasize that the formulas here result from a perturbation analysis of the full boundary value problem without any heuristic modelling assumptions or parameter fitting.

It has been found that the magnitude of the coefficient $\Lambda^{(\epsilon)} / 2 a$ is significantly larger than $\lambda^{(\theta)} / 2 a$ for many geometrical configurations; indeed the two can differ by an order of magnitude (cf.: figures 4 and 5). This evidence suggests that the effect on hydrodynamic slip of a weakly viscous subphase is likely to be far more important 
in most circumstances than any drag reduction/enhancement due to curvature of the meniscus. Our formulas provide a route to easy estimates on the balance between $\epsilon$ and $\theta$ at which the effects on slip should be of approximately equal significance.

From a broader perspective, we have demonstrated the usefulness of reciprocity ideas in calculating slip lengths in longitudinal channel flows over superhydrophobic surfaces when additional physical effects are introduced perturbatively. The same ideas can be used in several variants of this problem, including cases where different assumptions are made about the grooves (e.g. the case of 'dead-end' grooves wherein there is an induced pressure gradient required by a condition in steady state of a zero net flux condition) or the viscosity of the fluid in the subphase is large (compared to the working fluid), a situation pertinent to the study of so-called liquid-infused surfaces (Wexler, Jacobi \& Stone 2015). By way of example, the supplementary materials includes the analysis of the first-order slip length correction in the upper fluid in the high viscosity contrast limit $\epsilon \gg 1$.

\section{Acknowledgements}

D.G.C. is supported by an EPSRC Established Career Fellowship (EP/K019430/10), EPSRC grant EP/K041134/1 and a Royal Society Wolfson Research Merit Award.

\section{Supplementary material}

Supplementary material is available at https://doi.org/10.1017/jfm.2017.274.

\section{REFERENCES}

Asmolov, E. S. \& Vinogradova, O. I. 2012 Effective slip boundary conditions for arbitrary one-dimensional surfaces. J. Fluid Mech. 706, 108-117.

BAier, T., StefFes, C. \& HARDT, S. 2010 Thermocapillary flow on superhydrophobic surfaces. Phys. Rev. E 82, 037301.

Crowdy, D. G. 2010 Slip length for longitudinal shear flow over a dilute periodic mattress of protruding bubbles. Phys. Fluids 22, 121703.

Crowdy, D. G. 2016 Analytical formulas for longitudinal slip lengths over unidirectional superhydrophobic surfaces with curved menisci. J. Fluid Mech. 791, R7.

Davies, J., MaYnes, D., WebB, B. W. \& Woolford, B. 2006 Laminar flow in a microchannel with superhydrophobic walls exhibiting transverse ribs. Phys. Fluids 18, 087110.

Lauga, E., Brenner, M. P. \& Stone, H. A. 2007 Microfluidics: The No-slip Boundary Condition. Springer.

Lauga, E. \& Stone, H. A. 2003 Effective slip in pressure-driven Stokes flow. J. Fluid Mech. 489, 55-77.

MAYnes, D., JefFs, K., Woolford, B. \& WebB, B. W. $2007 a$ Laminar flow in a microchannel with hydrophobic surface patterned microribs oriented parallel to the flow direction. Phys. Fluids 19, 093603.

MAYnes, D., WebB, B. W. \& DAvies, J. $2007 b$ Laminar flow in a microchannel with hydrophobic surface patterned microribs oriented parallel to the flow direction. Phys. Fluids 19, 093603.

Neto, C., Evans, D. R., Bonaccurso, E., Butt, H.-J. \& Craig, V. S. J. 2005 Boundary slip in Newtonian liquids: a review of experimental studies. Rep. Prog. Phys. 68, 2859.

NG, C.-O., CHU, H. C. W. \& WANG, C. Y. 2010 On the effects of liquid-gas interfacial shear on slip flow through a parallel-plate channel with superhydrophobic grooved walls. Phys. Fluids 22, 102002.

NG, C.-O. \& WANG, C. Y. 2009 Stokes shear flow over a grating: implications for superhydrophobic slip. Phys. Fluids 21, 013602. 
Nizkaya, T. T. V., Asmolov, E. S. \& Vinogradova, O. I. 2013 Flow in channels with superhydrophobic trapezoidal textures. Soft Matt. 9, 11671-11679.

Nizkaya, T. V., Asmolov, E. S. \& Vinogradova, O. I. 2014 Gas cushion model and hydrodynamic boundary conditions for superhydrophobic textures. Phys. Rev. E 90, 043017.

Ou, J., Perot, B. \& Rothstein, J. P. 2004 Laminar drag reduction in microchannels using ultrahydrophobic surfaces. Phys. Fluids 16, 4635.

OU, J. \& Rothstein, J. P. 2005 Drag reduction and $\mu$-PIV measurements of the flow past ultrahydrophobic surfaces. Phys. Fluids 17, 103606.

PhILIP, J. R. 1972 Flows satisfying mixed no-slip and no-shear conditions. Z. Angew. Math. Phys. 23, 353-372.

Rothstein, J. P. 2010 Slip on superhydrophobic surfaces. Annu. Rev. Fluid Mech. 42, 89-109.

Sbragaglia, M. \& Prosperetti, A. 2007 A note on the effective slip properties for microchannel flows with ultrahydrophobic surfaces. Phys. Fluids 19, 043603.

SCHNitZER, O. 2016 Singular effective slip length for longitudinal flow over a dense bubble mattress. Phys. Rev. Fluids 1, 052101(R).

Schönecker, C., BAIER, T. \& HARDT, S. 2014 Influence of the enclosed fluid on the flow over a microstructured surface in the Cassie state. J. Fluid Mech. 740, 168-195.

SCHÖNECKER, C. \& HARDT, S. 2013 Longitudinal and transverse flow over a cavity containing a second immiscible fluid. J. Fluid Mech. 717, 376-394.

Solomon, B. R., Khalil, K. S. \& Varanasi, K. K. 2014 Drag reduction using lubricantimpregnated surfaces in viscous laminar flow. Langmuir 30, 10970-10976.

Squires, T. M. 2008 Electrokinetic flows of inhomogeneously slipping surfaces. Phys. Fluids 20, 092105.

TEO, C. J. \& KHOо, B. C. 2010 Flow past superhydrophobic surfaces containing longitudinal grooves: effects of interface curvature. Microfluid. Nanofluid. 9, 499-511.

WeXler, J. S., JAcobi, I. \& Stone, H. A. 2015 Shear-driven failure of liquid-infused surfaces. Phys. Rev. Lett. 114, 168301.

Woolford, B., MaYnes, D.\& WebB, B. W. 2009 Liquid flow through microchannels with grooved walls under wetting and superhydrophobic conditions. Microfluid. Nanofluid. 7, 121-135. 